\title{
A Layout Algorithm for Bar-Visibility Graphs on the Möbius Band
}

\author{
Alice M. Dean \\ Department of Mathematics and Computer Science \\ Skidmore College, Saratoga Springs, NY 12866 \\ adean@skidmore.edu, www.skidmore.edu/ ${ }^{\sim a d e a n}$
}

\begin{abstract}
We characterize two types of bar-visibility graphs on the Möbius band (abbreviated "BVGMs"), in which vertices correspond to intervals that are parallel or orthogonal to the axis of the band, depending on type, and in which adjacency corresponds to orthogonal visibility of intervals. BVGMs with intervals orthogonal to the axis are shown to be equivalent to the "polar visibility graphs" studied by Hutchinson [7]. BVGMs with intervals parallel to the axis are characterized as those graphs $G$ which satisfy the following conditions: $G$ is embedded on the Möbius band; the block-cutpoint tree of $G$ is a caterpillar in which all but at most one block is planar; and the non-planar block, if it exists, is at the "head" of the caterpillar.
\end{abstract}

\section{Introduction}

A bar-visibility graph in the plane (abbreviated $B V G P$ ) is a graph having a representation in which vertices correspond to disjoint, horizontal intervals ("bars") in the plane, with two vertices adjacent if and only if there is a vertical band of visibility between their bars, i.e., a non-degenerate rectangle with upper and lower sides contained in the bars representing the two vertices, and intersecting no other interval representing a vertex. Wismath [14], and independently, Tamassia and Tollis [10], characterized the graphs which can be represented as bar-visibility graphs in the plane as those graphs having a plane embedding with all cutpoints on a single face, and they gave linear-time algorithms to produce a layout of a bar-visibility graph.

Characterizations and algorithms have since been given for bar-visibility on other surfaces. In [12] BVGs on the cylinder (bars parallel to the axis of the cylinder) are considered, and in 11 spherical BVGs (which are equivalent to cylindrical BVGs with bars orthogonal to the axis) are considered. BVGs on the torus are considered in [9], and BVGs on the projective plane and the Klein bottle are studied in [7. In this paper we consider BVGs on the Möbius band, primarily with bars parallel to the axis of the band. We generalize the results of [12] to obtain a characterization and a linear-time layout algorithm for these graphs.

J. Marks (Ed.): GD 2000, LNCS 1984, pp. 350 359 2001.

(C) Springer-Verlag Berlin Heidelberg 2001 


\section{Parallel BVGs on the Cylinder}

In 12 Tamassia and Tollis characterize graphs having a visibility layout on a cylinder, with bars parallel to the axis of the cylinder, as those graphs which are planar and whose block-cutpoint tree is a caterpillar. We call such a layout a $B V G C P$ layout. A fundamental feature of their layout is that the underlying rectangle, whose top and bottom sides are identified to form a cylinder, is divided into rows and columns. There is one row for each vertex, and one column for each face of the embedding. Each vertex is represented by a horizontal interval in the appropriate row, and each face by a vertical interval in the appropriate column; edges are non-degenerate rectangles whose upper and lower borders are contained in the intervals corresponding to their endpoints, and whose left and right borders are contained in the intervals corresponding to their incident faces.

Cylindrical embeddings and their BVGCP layouts are easily seen to induce BVGMP embeddings and layouts, as indicated in the following proposition. An example of the induced Möbius embedding and layout is shown in Fig. 1

Proposition 1. Let $G$ be a graph with an embedding on the cylinder. Such an embedding induces an embedding of $G$ on the Möbius band. Furthermore, if the cylindrical embedding has a corresponding BVGCP layout (i.e., if the blockcutpoint tree of $G$ is a caterpillar), then that layout induces a BVGMP layout of $G$.
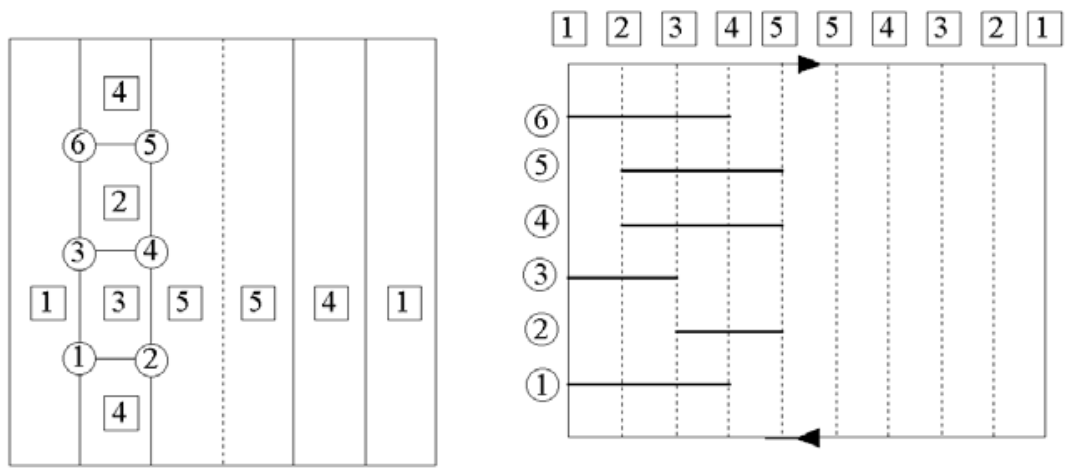

Fig. 1. A Möbius embedding and layout induced by a BVGCP

\section{Layout Algorithms for Parallel BVGMs}

In this section we generalize the algorithm of [12] to give a BVGMP layout for an arbitrary 2-connected graph embedded on the Möbius band. Our layout has two columns for each face of the embedding, arranged symmetrically around the 
vertical center line of a base rectangle for the Möbius band. The algorithm is somewhat more complex in this setting and requires some additional terminology.

Definition 1. 1. $R_{E}(G)$ denotes an embedding of a graph $G$ on a base rectangle $R_{E}$ for the Möbius band $M$, formed by identifying top and bottom borders of the rectangle $R_{E}$, in opposite directions.

2. A partial edge of $R_{E}(G)$ that intersects the top or bottom border of $R_{E}$ is called a "split edge." A split edge that extends from the top to the bottom of $R_{E}$ is called a "full-length" edge.

3. The base graph of $R_{E}(G)$, denoted $G_{b}$, is the plane graph induced by deleting all split edges.

4. If $R_{E}(G)$ has any split edges, then before identification of sides, there are distinct left and right faces, denoted $L$ and $R$. In the cylindrical identification of [12], the faces $L$ and $R$ remain distinct after identification, but in the Möbius identification they merge into a single face adjacent to the boundary of the band.

5. Two Möbius embeddings of a graph, $M_{1}(G)$ and $M_{2}(G)$, are called equivalent if they have equivalent base rectangle embeddings (i.e., embeddings with identical vertex rotation schemes, $c f$. [13] ). In particular, the faces of $M_{1}(G)$ and $M_{2}(G)$ that border the boundary of $M$ are identical.

6. A Möbius embedding $M(G)$ is called cylindrical if it is equivalent to the Möbius embedding induced by a cylindrical embedding $C(G)$ (cf. the proof of Prop. 1, cf. [2]); otherwise it is called non-cylindrical. Note that a planar graph may have a non-cylindrical embedding (for example, it is simple to provide such a drawing for $K_{4}$ ), but a non-planar graph does not have a cylindrical drawing, since all cylindrical graphs are planar.

Remark 1. We present the algorithm to obtain a BVGMP layout from an embedding $R_{E}(G)$ in four stages. For more details, see [2].

- Obtain a layout for a 2-connected graph $G$ with non-cylindrical embedding $R_{E}(G)$ such that $G_{B}$ is also 2-connected, and such that the border of the face $L$ contains 2 or more vertices;

- Obtain a layout for a 2-connected graph $G$ with non-cylindrical embedding $R_{E}(G)$ such that $G_{B}$ is also 2-connected, and such that the border of the face $R$ contains 2 or more vertices;

- Obtain a layout for a 2-connected graph $G$ with non-cylindrical embedding $R_{E}(G)$ such that $G_{B}$ is 1-connected but not 2-connected;

- Obtain a layout for an arbitrary graph $G$ with embedding $R_{E}(G)$.

Lemma 1. Let $P$ be a 2-connected plane graph, and let $S=<s_{1}, \ldots, s_{i}>$ and $T=<t_{1}, \ldots, t_{j}>$ be disjoint counterclockwise paths on the outer boundary cycle of $P$ (we think of $S$ as containing the "bottom" vertices of the cycle, and $T$ the "top" vertices). Given a sequence of $n$ numbers, $v_{1}<v_{2}<\ldots<v_{n}, P$ has an st-numbering (cf. [6]), with $s=s_{1}$ and $t=t_{j}$, in which the vertices of $S$ are numbered consecutively upward starting with $v_{1}$, and the vertices of $T$ are numbered consecutively upward ending with $v_{n}$. Furthermore, the numbers of the 
"left" vertices, namely those (if any) on the clockwise path from $s_{1}$ to $t_{j}$, have increasing numbers. Likewise, the "right" vertices, those on the counterclockwise path from $s_{i}$ to $t_{1}$ also have increasing numbers.

Definition 2. A numbering of a plane graph as described in Lemma1, is called a Möbius numbering of the graph. The base graph on the left in Fig. 圆 gives an example of a Möbius numbering, in which $S=\{1,2,3,4\}$ and $T=\{7,8,9,10\}$.

Algorithm 1. Input: A non-cylindrical embedding $R_{E}(G)$ of a graph $G$ on a base rectangle $R_{E}$ for the Möbius band $M$, such that the base graph $G_{b}$ is 2-connected (so in particular, $G$ is 2-connected), and such that the preidentification face $L$ has at least two vertices on it. Output: A parallel BVGM layout $R_{B}(G)$ for $\mathrm{G}$ on a base rectangle $R_{B}$ for $M$.

1. Construct a Möbius numbering for $G_{b}$ such that the top (resp. bottom) vertices of the numbering are those that are incident with the upper (resp. lower) half of a split edge. Then the left (resp. right) vertices of the numbering lie on the border of the face $L$ (resp. $R$ );

2. Give the edges of $G_{b}$ the orientation induced by the Möbius numbering of its vertices. $G_{b}$ is an acyclic digraph with the bottom and top vertices of $L$ as its unique source and sink. Complete this to an acyclic orientation of $R_{E}(G)$ by directing all split edges from bottom to top. Thus the borders of $L$ and $R$ are both oriented bottom to top;

3. Let $D^{*}$ be the digraph whose vertices correspond to the faces of $R_{E}(G)$, including the internal faces, the faces $L$ and $R$, and the upper half $F_{T}$ of each split face $F$. (Note that we may assume that each split face has exactly one upper and one lower half, and that the two halves are distinct, cf. [2].) The edges of $D^{*}$ are the dual edges of $R_{E}(G)$, including upper split edges but not lower split edges, and are directed from left to right across the oriented edges of $R_{E}(G) . D^{*}$ is an acyclic digraph with the faces $L$ and $R$ as its unique source and sink;

4. The vertices of $D^{*}$ are given a topological numbering corresponding to the orientation of $D^{*}$, and the lower half $F_{B}$ of each split face is given the same number as its upper half. $L$ receives the lowest number, $R$ the highest, and the upper half-faces and $R$ are numbered sequentially left to right. Each number is also given a subscript: subscript $I$ (for "Alg. 11') for $L, R$, and all internal faces; $T$ (for "top") for all upper half-faces; and $B$ (for "bottom") for all lower half-faces.

5. Construct a base rectangle $R_{B}$ for the BVGMP layout, with $n+2$ rows and $2 m$ columns, where $n$ is the number of vertices of $G$, and $m$ is the number of faces in the embedding $M(G)$ on the Möbius band $M$ that is induced by $R_{E}(G)$. The bottom and top rows are not used for vertices; the remaining rows are numbered bottom to top in increasing order, using the Möbius numbering of $G_{b}$. The first $m$ columns are numbered left to right in increasing order, using the subscripted, topological numbering of the left, internal, and upper faces; the next $m-1$ columns are numbered left to right in decreasing order, using the lower and internal faces, but the subscript $I$ 
for each internal face is changed to $I I$ (for "Alg. 22', which makes use of these columns); the last column is given the $I$-subscripted number of $R$. Thus each vertex corresponds to one row, and each face to two columns, symmetrically arranged around the centerline of $R_{B}$, and no two columns have the same subscripted number.

6. Construct an interval to represent each $v \in G$. Let Left $(v)($ resp. Right $(v)$ ) denote the number of the face in $R_{E}(G)$ to the left of the leftmost incoming and outgoing edges (resp. to the right of the rightmost incoming and outgoing edges). The interval for $v$ is placed in the row having the number of $v$, and it extends from column Left $(v)$ to column $\operatorname{Right}(v)$.

The resulting layout on $R_{B}$ is equivalent to the embedding $R_{E}(G)$.

Example 1. The left-hand diagram in Fig. 2 shows an embedding of the Petersen graph on a base rectangle for the Möbius band, and the right-hand diagram shows the results of applying Algorithm 1 to help readability, the dual edges are not shown. The BVGMP layout that results is shown on the right in Fig. 2.
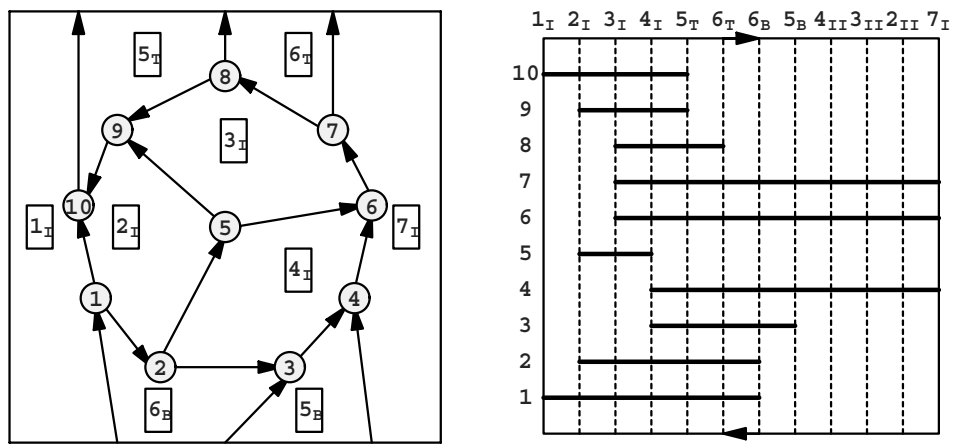

Fig. 2. Application of Algorithm 1 to the Petersen graph

We assume without loss of generality that both half-faces $L$ and $R$ contain one or more vertices, and that one or the other contains two or more vertices (cf. [2]). Algorithm 1 requires that the left-hand face $L$ contain two or more vertices, so that the source and sink vertices are, respectively, at the bottom and top of $L$. If $L$ does not have two vertices on its border, but $R$ does, it is a simple matter to first reflect the embedding over the vertical center line of the base rectangle, exchanging the roles of $L$ and $R$. However, when we extend the algorithm to the case in which the base graph $G_{b}$ is not 2-connected, we want to use the same labels on all faces, regardless of which case each block falls into. Algorithm 11 can easily be modified so that a reflection of the embedding is not required. Details of the algorithm are omitted, but the essential idea is to lay out each vertex interval from right to left, using the column labels with subscript $I I$ rather than 
$I$, and with $1_{I}=(m+1)_{I I}$ and $(m+1)_{I}=1_{I I}$, where $m+1$ is the number of faces on $M$.

Algorithm 2. Input: A non-cylindrical embedding $R_{E}(G)$ of a graph $G$ on a base rectangle $R_{E}$ for the Möbius band $M$, such that the base graph $G_{b}$ is 2connected, and such that the pre-identification face $R$ has at least two vertices on it. Output: A parallel BVGM layout $R_{B}(G)$ for $\mathrm{G}$ on a base rectangle $R_{B}$ for $M$.

Algorithms 10 and 2 give a BVGMP layout for a graph embedded on the Möbius band, if the base graph $G_{b}$ is 2-connected. In the next algorithm we handle the case when $G$ is 2 -connected but $G_{b}$ is not. We make the layout by filling in the bars one block at a time. First we need another definition.

Definition 3. Suppose that $R_{E}(G)$ is an embedding of the 2-connected graph $G$ on a base rectangle for Möbius band, with base graph $G_{b}$ that is connected but not 2-connected, and let $B$ be a block of $G_{b}$. We order the external faces (i.e., the upper and lower split faces, and $L$ and $R$ ) from left to right and top to bottom, so that $L<$ any upper split face $<$ any lower split face $<R$. We denote by Left $(B)$ (resp., Right $(B)$ ) the first (resp., last) external face, in this ordering, that contains on its border some edge or split edge incident with $B$ (such an edge may connect a vertex in $B$ to another not in $B$ ).

As an example, in the embedding at the upper left of Fig. 3, consider the $G_{b}$-block $B$ containing the vertices $v$ and $y . \operatorname{Left}(B)=L$, and $\operatorname{Right}(B)$ is the middle lower split face. A key observation is that, when we lay out each block of $G_{b}$, no adjacency involving an edge of $B$ is represented by visibilities outside the columns from $\operatorname{Left}(B)$ to $\operatorname{Right}(B)$. If a vertex is a cutpoint of $G$, then its bar is a composite of the bars from the blocks to which it belongs.

Algorithm 3. Input: A non-cylindrical embedding $R_{E}(G)$ of a graph $G$ on a base rectangle $R_{E}$ for the Möbius band $M$, such that the base graph $G_{b}$ is connected but not 2-connected, and such that $L$ and $R$ are the only external faces touching both the top and bottom sides of the base rectangle. Output: A parallel BVGM layout $R_{B}(G)$ for $\mathrm{G}$ on a base rectangle $R_{B}$ for $M$.

1. The layout is done on a rectangle $R_{B}(G)$ with $2 m$ columns and $n+2$ rows, as in Algs. 1 and 2. The column labels are the same as before (with the first and the last columns doubly labeled for use in either Alg. 1 or Alg. 2). The row-labels are determined in subsequent steps, as each block is laid out.

2. The external faces of $R_{E}(G)$ are numbered as in Algs. 1 and 2. The numbers on the internal faces are assigned as each block is laid out. The blocks are laid out one by one, in the order of a breadth-first traversal of the block-cutpoint tree of $G_{b}$, by executing steps 3 to 6 for each block $B$.

3. Determine the values of $\operatorname{Left}(B)$ and $\operatorname{Right}(B)$; the bars of $B$ are contained between the corresponding columns in the layout.

4. Contract the vertices of all blocks other than $B$, so that only the vertices of $B$ remain; call the resulting embedded graph $E(B)$. At this point there may be multiple edges or loops at vertices of $B$ that were cutpoints of $G$. If 
neither face $L$ nor $R$ of $E(B)$ has two or more vertices on the border, then delete multiple split edges or split loops until that is the case. These edges are handled in Step 6.

5. Use either Alg. 1 or Alg. 2 to number and orient $E(B)$ and its dual. If a cutpoint of $B$ was numbered in a previous step, the same number must be used now as well. For non-cutpoints, use numbers that have not yet been used, chosen so that a Möbius-numbering results. For internal faces of $E(B)$, use the lowest possible face-numbers that have not yet been used. (The reason that the block-cutpoint tree is traversed in breadth-first order is to avoid vertex-numbering conflicts in this step; it doesn't matter what particular numbers are used, so long as they obey the rules for Möbius numbering.)

6. If any edges were deleted in Step 4 put them back now and orient them from bottom to top. For each vertex $v$ in $B$, let $\operatorname{Left}_{B}(v)$ be either $\operatorname{Left}(v)$ in $E(B)$ or Left $(B)$, whichever is further right. Similarly, $\operatorname{Right}_{B}(v)$ is either $\operatorname{Right}(v)$ in $E(B)$ or $\operatorname{Right}(B)$, whichever is further left. In other words, we "trim" the bar for $v$ so that it is contained in the region from $\operatorname{Left}(B)$ to $\operatorname{Right}(B)$.

7. Once all the blocks of $G$ have been labeled, we draw the bars for the layout of $G$. There is one row for each vertex, in increasing order of vertex numbers. The bar for a vertex $v$ is the union of the bars from $\operatorname{Left}_{B}(v)$ to $\operatorname{Right}_{B}(v)$, for each block $B$ containing $v$. The final result is a BVGMP layout for $G$.

Example 2. Figure 3illustrates several steps of Algorithm 3. as well as the final layout. Blocks $B_{1}$ and $B_{2}$ are shown with other blocks contracted, and captions indicate which of Algorithms 1 and 2 is used, as well as the values of $\operatorname{Left}\left(B_{i}\right)$ and $\operatorname{Right}\left(B_{i}\right)$ (these values are computed before contracting the other blocks). Partial edges that were deleted in Step 4, and then put back in Step 6, are indicated by dotted lines.

Note that if an embedding $R_{E}(G)$ has $G_{b}$ disconnected, then it is easy to construct a cylindrical embedding for $G$. Hence, if $G$ is non-cylindrical and 2connected, then $G_{b}$ is connected. This, together with Algorithms 12 and 3 . gives the following theorem.

Theorem 1. If $G$ is a 2-connected graph embedded on the Möbius band, then there is an equivalent parallel BVGM layout of $G$.

\section{Characterizations of Parallel and Orthogonal BVGMs}

In 12 Tamassia and Tollis show that a planar graph $G$ admits a parallel BVG layout on the cylinder if and only if its block-cutpoint tree is a caterpillar. In this section we give an analogue of that result for parallel BVG layouts on the Möbius band. Now that we have established an algorithm for 2-connected graphs, the remaining results of [12] generalize quite straightforwardly (cf [2]). 
Definition 4. If the block-cutpoint tree $T$ of a graph $G$ is a caterpillar, then it is easy to see that the caterpillar's central path can be taken to be of the form $P=c_{0}-B_{1}-c_{1}-B_{2}-\ldots-c_{(k-1)}-B_{k}-c_{k}$, where the $c_{i}$ are all the cutpoints of $G$, and the $B_{i}$ are the non-leaf blocks of $T$. Any additional blocks are leaf-blocks, each containing a single cutpoint. We call this central path $P$ the spine of $T$, and we call any leaf-block incident with either $c_{0}$ or $c_{k}$ head-block of $T$.
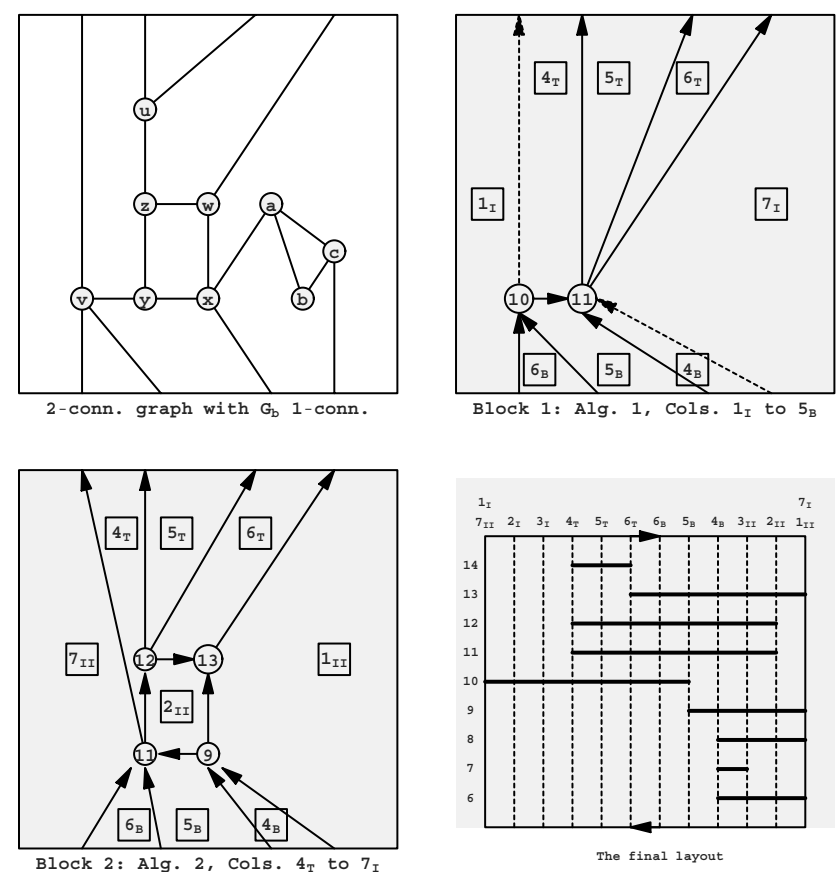

Fig. 3. Applying Algorithm 3 to a 2-connected graph

Theorem 2. A graph $G$ has a parallel BVG layout on the Möbius band if and only if all the following conditions hold: (1) The block-cutpoint tree $T$ of $G$ is a caterpillar; (2) At most one block of $G$ is non-planar; and (3) The non-planar block B, if it exists, must be a head-block in $T$.

One can also consider BVGMs with bars orthogonal to the axis of the band. It is easy to show that these are equivalent to the polar and circular visibility graphs (abbreviated PVGs and CVGs) that are characterized by Hutchinson in [7. These are graphs with vertices represented by circular arcs in the projective plane. In CVGs arcs are permitted to be complete circles, while in PVGs they are not. There is a reversible transformation taking CVGs and PVGs to orthogonal BVGMs, yielding the following equivalences. Characterizations of CVGs and BVGs, hence also of orthogonal BVGMs, can be found in [7]. 
Theorem 3. A graph $G$ is a $C V G$ if and only if it is an orthogonal BVGM. $G$ is a PVG if and only if it is an orthogonal BVGM in which bars may not be complete circles.

\section{Concluding Remarks and Acknowledgments}

It would be interesting to find applications of visibility representations on the Möbius band in VLSI design or elsewhere, since the class of BVGMPs includes the class of BVGCPs. It should also be possible to extend these methods to higher-genus, non-orientable surfaces. Another visibility class that has been studied is rectangle-visibility graphs (RVGs), in which vertices are represented by rectangles, and edges by visibility in both the vertical and horizontal directions. While a number of papers have been published on RVGs in the plane [3/48], very little has been done on RVGs on higher-genus surfaces. Hutchinson and the author 5 have obtained edge-bounds for RVGs on the cylinder and torus.

This paper was written while the author was on sabbatical in England in 1999. She wishes to express her gratitude to the members of the School of Mathematical and Information Sciences at Coventry University for their warm welcome and generosity while she was a visitor there. She is also grateful to Joan Hutchinson and Tom Shermer for several helpful conversations.

\section{References}

1. G. Di Battista, P. Eades, R. Tamassia, and I. G. Tollis. Graph Drawing: Algorithms for the visualization of graphs. Prentice Hall, Upper Saddle River, NJ, 1999.

2. A. M. Dean. Bar-visibility graphs on the Möbius band. Manuscript, 1999.

3. A. M. Dean and J. P. Hutchinson. Rectangle-visibility representations of bipartite graphs. Discrete Applied Math., 75:9-25, 1997.

4. A. M. Dean and J. P. Hutchinson. Rectangle-visibility of unions and products of trees. J. Graph Algorithms and Applications, 2:1-21, 1998.

5. A. M. Dean and J. P. Hutchinson. Rectangle-visibility graphs on surfaces. Manuscript, 1999.

6. S. Even and R. E. Tarjan. Computing an st-numbering. Theoret. Comput. Sci., 2:339-344, 1976.

7. J. P. Hutchinson. On polar visibility representations of graphs. In: Proc. Graph Drawing 2000, J. Marks, ed., to appear.

8. J. P. Hutchinson, T. Shermer, and A. Vince. On representations of some thicknesstwo graphs (extended abstract). In: Lecture Notes in Computer Science \#1027, F. Brandenburg, ed., Springer, Berlin, 159-166, 1995.

9. B. Mohar and P. Rosenstiehl. Tessellation and visibility representations of maps on the torus. Discrete Comput. Geom., 19:249-263, 1998.

10. R. Tamassia and I. G. Tollis. A unified approach to visibility representations of planar graphs. Discrete Comput. Geom., 1:321-341, 1986.

11. R. Tamassia and I. G. Tollis. Tessellation representations of planar graphs. In: Proc. 27th Annual Allerton Conf., University of Illinois at Urbana-Champaign, 48-57, 1989. 
12. R. Tamassia and I. G. Tollis. Representations of graphs on a cylinder. SIAM J. Disc. Math., 4:139-149, 1991.

13. A. White. Graphs, Groups and Surfaces (revised ed.). North-Holland, Amsterdam, 1984.

14. S. K. Wismath. Characterizing bar line-of-sight graphs. In: Proc. 1st ACM Symp. Comput. Geom., 147-152, 1985. 